\title{
AN INVARIANT ON 3-DIMENSIONAL LIE ALGEBRAS
}

\author{
HIROYUKI TASAKI AND MASAAKI UMEHARA
}

(Communicated by Palle E. T. Jorgensen)

\begin{abstract}
We construct an extra symmetric bilinear form on a 3-dimensional Lie algebra $\mathfrak{g}$ which induces an invariant $\chi(\mathfrak{g})$ on $\mathfrak{g}$. Moreover it provides a new viewpoint for the classical classification of 3-dimensional Lie algebras
\end{abstract}

In this note, we shall construct an extra symmetric bilinear form $S$ on a 3-dimensional Lie algebra, which provides new viewpoints for the classical classification of 3-dimensional Lie algebras.

Let $\mathfrak{g}$ be a 3-dimensional Lie algebra with the Lie bracket [ , ] over a field $k$ of characteristic $\neq 2$. Let $\left\{e_{1}, e_{2}, e_{3}\right\}$ be a fixed basis. There is a canonical identification $\wedge^{2} \mathfrak{g}^{*} \cong \mathfrak{g}$ by $e_{1}=e_{2}^{*} \wedge e_{3}^{*}, e_{2}=e_{3}^{*} \wedge e_{1}^{*}$, and $e_{3}=e_{1}^{*} \wedge e_{2}^{*}$, where $\left\{e_{1}^{*}, e_{2}^{*}, e_{3}^{*}\right\}$ is the dual basis of $\mathfrak{g}^{*}$ with respect to $\left\{e_{1}, e_{2}, e_{3}\right\}$. Then the bracket $[,] \in\left(\Lambda^{2} \mathfrak{g}^{*}\right) \otimes \mathfrak{g}$ is considered as an element of $\mathfrak{g} \otimes \mathfrak{g}$ by the identification and induces a bilinear form $L: \mathfrak{g}^{*} \times \mathfrak{g}^{*} \rightarrow k$, which is invariant under the change of a basis up to scalar multiplications in $k$. If we set the structure constants of the bracket $[$,$] by$

$$
\begin{aligned}
& {\left[e_{2}, e_{3}\right]=a_{11} e_{1}+a_{12} e_{2}+a_{13} e_{3},} \\
& {\left[e_{3}, e_{1}\right]=a_{21} e_{1}+a_{22} e_{2}+a_{23} e_{3},} \\
& {\left[e_{1}, e_{2}\right]=a_{31} e_{1}+a_{32} e_{2}+a_{33} e_{3},}
\end{aligned}
$$

then the representation matrix of $L$ with respect to the basis $\left\{e_{1}^{*}, e_{2}^{*}, e_{3}^{*}\right\}$ is written by $A=\left(a_{i j}\right)_{i, j=1,2,3}$. If we change the basis on $\mathfrak{g}$ by a matrix $P=\left(p_{i j}\right)_{i, j=1,2,3} \in \mathrm{GL}(3, k)$ such that $e_{j}^{\prime}=\sum_{i=1}^{3} p_{i j} e_{i}$, then new structure constants $A^{\prime}$ are given by $A^{\prime}=(\operatorname{det} P) P^{-1} A^{\mathrm{t}} P^{-1}$.

Now, we define another bilinear form $S: \mathfrak{g} \times \mathfrak{g} \rightarrow k$ by

$$
S(u, v)=L\left(u_{1}^{*}, v_{1}^{*}\right) L\left(u_{2}^{*}, v_{2}^{*}\right)-L\left(u_{1}^{*}, v_{2}^{*}\right) L\left(u_{2}^{*}, v_{1}^{*}\right) \quad \text { for } u, v \in \mathfrak{g},
$$

where $u=u_{1}^{*} \wedge u_{2}^{*}$ and $v=v_{1}^{*} \wedge v_{2}^{*}$ with respect to the identification $\wedge^{2} \mathfrak{g}^{*} \cong \mathfrak{g}$. Then it can be easily checked that the representation matrix of $S$ coincides with the cofactor matrix $A^{*}$ of $A$. Since $A^{\prime *}={ }^{t} P A^{*} P$, the bilinear form $S$ is determined independently of the choice of a basis. The following lemma is immediately obtained from the Jacobi identity.

Received by the editors October 3, 1990.

1991 Mathematics Subject Classification. Primary 17B05; Secondary 17B30.

Key words and phrases. 3-dimensional Lie algebra. 
Lemma. The bilinear form $S$ is symmetric, namely, ${ }^{\mathrm{t}} A^{*}=A^{*}$.

It should be remarked that $\mathfrak{g}$ is unimodular if and only if the matrix $A$ is symmetric. When $k$ is algebraically closed, the isomorphism classes of 3dimensional unimodular Lie algebras are classified by the rank of the matrix $A$.

Theorem 1. Let $\mathfrak{g}$ be a 3-dimensional Lie algebra. Then the bilinear form $S$ defined by (2) is proportional to the Killing form $F$ of $\mathfrak{g}$.

Proof. Let $B$ be a representation matrix of the Killing form $F$. By a straightforward calculation, one can obtain the identity $B=\hat{A}^{*}-2 A^{*}$, where $\hat{A}^{*}$ is the cofactor matrix of $\hat{A}=A-{ }^{t} A$. If $\mathfrak{g}$ is unimodular, then $\hat{A}^{*}=0$ and $F=-2 S$ holds. So we may assume that $\mathfrak{g}$ is not unimodular. Then $\mathfrak{g}$ is solvable and the basis $\left\{e_{1}, e_{2}, e_{3}\right\}$ can be chosen such that $a_{3 i}=a_{i 3}=0(i=1,2,3)$ (see $\left[1\right.$, p. 12]). Then one can easily verify that $\hat{A}^{*}-2 A^{*}$ is proportional to $A^{*}$. This proves the theorem.

By the theorem, we can define an invariant $\chi(\mathfrak{g}) \in \mathbb{P}=k \cup(\infty)$ by $F=$ $(\chi(\mathfrak{g})-2) S$, unless $F=S=0$; namely, $\mathfrak{g}$ is neither Heisenberg nor abdelian. There is another exceptional Lie algebra denoted by $\mathfrak{k}$, which is characterized by the property that the matrix $A$ is skew symmetric. One can easily verify that the well-known classification theorem (e.g., [1, p. 13; 2, Lemma 4.10]) of 3-dimensional Lie algebras is rewritten in the following

Theorem 2. Let $\mathfrak{g}$ be a 3-dimensional Lie algebra that is neither unimodular nor isomorphic to $\mathfrak{k}$. Then there exists a basis $\left\{e_{1}, e_{2}, e_{3}\right\}$ of $\mathfrak{g}$ such that

$$
\left[e_{3}, e_{2}\right]=e_{1}, \quad\left[e_{3}, e_{1}\right]=-e_{1}+\frac{1}{\chi(\mathfrak{g})} e_{2} \quad \text { and } \quad\left[e_{1}, e_{2}\right]=0
$$

\section{REFERENCES}

1. N. Jacobson, Lie algebras, Intersci. Tracts in Pure Appl. Math., vol. 10, John Wiley-Sons, New York, 1962.

2. J. Milnor, Curvature of left invariant metrics on Lie groups, Adv. in Math. 21 (1976), 293329.

Institute of Mathematics, University of Tsukuba, Tsukuba, Ibaraki 305, Japan

Department of Mathematics, College of General Education, Osaka University, ToyONAKA, OSAKA, 560, JAPAN

Current address: Masaaki Umehara: Department of Mathematics, College of General Education, Osaka University, Toyonaka, Osaka, 560 Japan

E-mail address: Hiroyuki Tasaki: a906023@sakura.cc.tsukuba.ac.jp 